Acta Crystallographica Section D

Biological

Crystallography

ISSN 0907-4449

\section{A. Echalier, ${ }^{a}$ R. L. Glazer, V. Fülöp ${ }^{a}$ and M. A. Geday ${ }^{b} *$}

${ }^{\text {a } U n i v e r s i t y ~ o f ~ W a r w i c k, ~ D e p a r t m e n t ~ o f ~}$ Biological Sciences, Gibbet Hill Road, Coventry CV4 7AL, England, and ${ }^{\mathbf{b}}$ Oxford Cryosystems Ltd, 3 Blenheim Office Park, Long Hanborough OX29 8LN, England

Correspondence e-mail:

m.geday@oxcryo.ferrarisgroup.com

\title{
Assessing crystallization droplets using birefringence
}

In this paper, the detection of crystalline elements in protein crystallization droplets containing precipitate is illustrated using the rotating-polarizer microscope technique. The sensitivity of this automated birefringence technique enables the detection of microcrystals in a precipitate that appears to be amorphous using traditional methods of inspection. The technique is illustrated with lysozyme and glucose isomerase. Glucose isomerase microcrystals were used successfully for seeding experiments and the conditions of both of the systems were refined to produce crystals suitable for X-ray analysis. The results are relevant to the field of high-throughput crystallography as an automated crystal-detection method as well as being a useful tool for detailed precipitate analysis.

\section{Introduction}

Finding crystallization conditions is often the most timeconsuming step in protein crystallography. Therefore, it is of the greatest importance to be able to detect the presence of any crystalline material in a precipitate. The standard procedure in the search for a protein crystallization condition is to use a sparse-matrix sampling experiment (for a review of crystallization techniques see, for example, Ducruix \& Giegé, 1999). This approach seeks to reduce the time needed for finding crystallization conditions. The design of these experiments is based on a statistical approach in which the crystallization conditions found most frequently in the literature are represented (Jancarik \& Kim, 1991). Crystallization screening experiments may or may not result in one or more conditions that lead to the detectable growth of protein crystals. In the former case, the crystallization conditions are refined until crystals suitable for X-ray analysis are obtained. However, in the latter case, if protein crystals are not obtained nor crystalline precipitate detected after careful examination of the precipitate, the protein and the effort is lost and very little information can be derived from the failed screening experiments. The next step in the quest for crystallization conditions will then typically be the setting up of a different screen and/or a laborious exploration of the phase-diagram space by varying the available crystallization parameters (such as precipitant, buffer, $\mathrm{pH}$, temperature, protein concentration, use of additives etc.). Consequently, it is very important to detect any microcrystals in the precipitate.

In this paper, we describe the use of a rotating-polarizer microscope technique (MetriPol) to detect protein crystallites in crystallization droplets as a part of a programme to devise an automatic tool for detecting crystals as well as promising crystallization conditions.

We have focused on two different proteins, glucose isomerase and lysozyme, as the two proteins have different
C 2004 International Union of Crystallography

Printed in Denmark - all rights reserved
Received 24 November 2003 Accepted 9 February 2004 
biochemical characteristics (molecular weight, pI, oligomeric state etc.). Both proteins are commercially available and their phase diagrams are well known and documented (Ducruix \& Giegé, 1999; Carrell et al., 1984).

\section{Optics theory and birefringence}

\subsection{Birefringence}

Throughout the history of protein crystallography, polarizing microscopy has been a tool regularly used by crystallographers to detect and characterize crystals prior to X-ray analysis. Partly because of the attractive colours produced, it is still one of the most used techniques for presenting crystal growth and habitat (Nollert, 2003). Polarizing microscopy depends on a sample being birefringent, i.e. the refractive index of light passing through the sample varies with the polarization of the incoming light. A linearly polarized light wave travelling along a general direction and polarized in a non-specific plane will, upon passing through a birefringent sample, become resolved into a faster and a slower travelling wave. These waves will emerge with a phase difference, $\delta$, depending on the magnitude of the plano-birefringence, $\Delta n_{p}$, the thickness of the sample, $L$, and the wavelength of the light, $\lambda$,

$$
\delta=(2 \pi / \lambda) \Delta n_{p} L
$$

The phase difference will generally make linearly polarized light elliptically polarized. Birefringence is seen in any optically anisotropic section of the sample. However, if the incoming light travels along an optically isotropic direction (an optical axis) or is polarized along either the fast or the slow polarization direction within the sample (extinction angles), the polarization of the light will remain unaltered. ${ }^{\mathbf{1}}$

Strictly speaking, the birefringence $\Delta n$ is a property characterizing the optical anisotropy of an object and is given by the difference in refractive indices along the principal axes of the refractive index surface (the indicatrix; Hartshorne \& Stuart, 1969). The observed optical anisotropy, the planobirefringence, on the other hand, depends on the direction of view and is therefore often not along one of the principal axes.

In crystal optics, crystals are grouped into three categories: isotropic crystals, uniaxial crystals and biaxial crystals which, apart from a few special cases, correspond to cubic pointgroup symmetry, trigonal, tetragonal or hexagonal pointgroup symmetry, and orthorhombic, monoclinic and triclinic point-group symmetry, respectively.

\subsection{Form birefringence}

The intrinsic birefringence resulting from the crystal structure or strain may be too weak to be detected. However, in samples consisting of thin layers or needles the phenomenon of form birefringence (Bragg \& Pippard, 1953; Oldenbourg \& Ruiz, 1989), $\Delta n_{f}$, will in some cases aid the detection of order and thus crystals or crystal precursors present in a precipitate.

\footnotetext{
${ }^{\mathbf{1}}$ We neglect any optically rotary effect (circular birefringence) since this effect is generally much weaker than the linear birefringence.
}

With a few approximations, the magnitude of $\Delta n_{f}$ can in the case of stacked platelets be cast as (Bragg \& Pippard, 1953)

$$
\Delta n_{f}=-\left(n_{c}-n_{l}\right) f \frac{9 k}{(3+k)^{2}},
$$

where $n_{c}$ and $n_{l}$ are the refractive indices of the crystal and the surrounding media, respectively, $f$ is the fractional volume occupied by the crystals and

$$
k=(1-f)\left(n_{c}^{2} / n_{l}^{2}-1\right) .
$$

For needles or rods (2) becomes

$$
\Delta n_{f}=\frac{1}{2}\left(n_{c}-n_{l}\right) f \frac{9 k}{(3+k)^{2}} .
$$

$\Delta n_{f}$ in (2) and (4) is no longer the birefringence of the crystals per se, but the birefringence of transparent objects suspended in the crystallization buffer. Thus, $\delta$ depends on the light path through the volume of the entire crystal suspension, increasing $L$ significantly.

Random stacking of platelets or packing of rods will have an optically uniaxial character, with the optical axis coinciding with the platelet normals or the directors of the rods, and thus we may approximate (1) for a uniaxial form-birefringent object by

$$
\delta=\frac{2 \pi}{\lambda} \Delta n_{p} L^{\prime} \simeq \frac{2 \pi}{\lambda} \sin ^{2}(\Theta) \Delta n L^{\prime}=\frac{2 \pi}{\lambda} \sin ^{2}(\Theta) \Delta n_{f} L^{\prime} .
$$

$\Theta$ is the angle between the optic axis in the sample and the direction of view and $L^{\prime}$ is the full light path through the suspension of ordered objects.

\subsection{Polarizing microscopy}

Classically, birefringence has been measured using the technique of crossed polars, where the sample is placed between two perpendicularly aligned polarisers: thus light will only be transmitted for areas of the sample where the polarization has been changed. The intensity expression for crossed polars, which can be found in most optics textbooks, is

$$
I=I_{0} \sin ^{2}(\delta / 2) \sin ^{2} 2(\alpha-\varphi) .
$$

Here, $\alpha-\varphi$ is the angle between the slow polarization orientation, 'the slow axis', inside the sample (at an angle $\varphi$ ) and the polariser (at an angle $\alpha$ ) and $I_{0}$ is the intensity of the light transmitted by the sample. ${ }^{2}$

In thin samples or samples with very low birefringence, the $\sin ^{2}(\delta / 2)$ term in (6) is very small. Thus, it can be difficult to detect the birefringence, since the sample, if oriented in a favourable orientation $\left[\sin ^{2}(\alpha-\varphi)>>0\right.$ ], will appear dark grey against a black background or as a slight colour change if retardation plates are used. If the sample is not favourably oriented $\left[\sin ^{2}(\alpha-\varphi) \simeq 0\right]$, the sample may not be detected at all.

Therefore, there is a need for a more sensitive detection method which furthermore is insensitive to the orientation of

\footnotetext{
${ }^{2}$ The refractive index is the ratio between the speed of light in the medium and the speed of light in vacuum $(n=c / v)$; hence, the slow axis is characterized by a refractive index larger than that of the fast axis.
} 
the sample. Here, we use the rotating-polarizer technique, where a rotating linear polarizer is combined with a circular analyser, consisting of a quarter-wave retarder plate and a linear analyser oriented at $45^{\circ}$ to one another. With this arrangement, called the MetriPol microscope (Glazer et al., 1996.; Geday et al., 2000; http://www.metripol.com), the intensity varies with the polarizer angle according to

$$
I=\frac{1}{2} I_{0}[1+\sin 2(\alpha-\varphi) \sin \delta] .
$$

This formula lends itself to numerical analysis when a computer-controlled rotating polarizer is used with a CCD camera.

The technique relies on capturing a series of images and performing a least-squares fit of the parameters $I_{0},|\sin \delta|$ and $\varphi$ to (7). This can, when rotating the polarizer through a number of equally spaced angular positions, be performed using simple Fourier analysis when casting (7) as

$$
I_{i} / I_{0}=a_{0}+a_{1} \sin 2 \alpha+a_{2} \cos 2 \alpha,
$$

where $a_{0}=\frac{1}{2}, a_{1}=\cos 2 \varphi \sin \delta$ and $a_{2}=-\sin 2 \varphi \sin \delta$.

Another advantage, apart from the increased sensitivity and the flexibility of the sample alignment, in using a technique such as MetriPol microscope is that the three components of birefringence in (7) are imaged separately in three artificially coloured images as follows.

(i) The transparency image $\left[I_{O}\right.$ in (7)], which corresponds to a black-and-white image as seen in non-polarized microscopy.

(ii) The $|\sin \delta|$ image, which illustrates the magnitude of the birefringence.

(iii) The $\varphi$ image showing the extinction angles, in this study the slow axis. We define $\varphi$ to be zero when the slow axis is horizontal and increasing in anticlockwise direction.

The birefringence information can be presented either as pure colour images ('topographies') or can be overlaid as azimuths, showing the $\varphi$ information by the inclination and the relative $|\sin \delta|$ value by the length. The azimuthal values are typically averaged over a square of $25 \times 25$ pixels.

In this study, we have used the intensity image to compare the birefringence results with what would have been observed in a standard microscope. We have used $|\sin \delta|$ information to identify areas of birefringence and the distribution of the slow axis (the $\varphi$ images) to analyse the physical character of the birefringent structures detected.

\section{Materials and methods}

\subsection{Protein preparation}

Glucose isomerase, kindly provided by Bob Cudney, Hampton Research, was resuspended, dialysed twice in $0.01 M$ HEPES pH 7.0 and concentrated to $68 \mathrm{mg} \mathrm{ml}^{-1}$ using Microcon concentrating units (Millipore) and filtered using $0.2 \mu \mathrm{m}$ filters. Hen egg-white lysozyme was purchased as a microcrystalline powder (Calbiochem) and was dissolved in $0.04 \mathrm{M}$ sodium acetate $\mathrm{pH} 4.7$ to a concentration of 45 or $75 \mathrm{mg} \mathrm{ml}^{-1}$. Prior to crystallization, the protein solutions were centrifuged at $13200 \mathrm{rev} \mathrm{min}^{-1}$ for $10 \mathrm{~min}$ at $277 \mathrm{~K}$.

\subsection{Crystallization}

The crystallization droplets were composed of an equal volume of the protein and precipitating solutions.

For glucose isomerase, the precipitating solutions were $0.05 M$ sodium acetate buffer $\mathrm{pH} 5.2$ with concentrations of ammonium sulfate varying from 3.8 to $0.1 \mathrm{M}$. For lysozyme, the precipitating solutions were $0.1 \mathrm{M}$ sodium acetate buffer $\mathrm{pH} 4.7$ with concentrations of sodium chloride varying from 4.0 to $0.3 M$. All crystallization experiments were carried out at $291 \mathrm{~K}$.

The crystallization trials were set up as hanging or sandwiched drops using vapour diffusion for equilibration. The hanging drops were mounted on siliconized glass cover slips on standard 24-well VDX plates (Molecular Dimensions) to enable transfer onto glass microscope slides with spacers where the MetriPol assessment was made. Sandwiched droplets were mounted on specially designed single wells between two siliconized cover slips, so that the birefringence measurements could be made in situ.

The imaging could not be performed on the VDX plates, since these were intrinsically birefringent.

\subsection{Image acquisition with MetriPol}

The images presented here were all captured using 50 different polarizer angles (i.e. $3.6^{\circ}$ steps; total of $180^{\circ}$ ) to give a high signal-to-noise ratio, although in practice faster $(\sim 8 \mathrm{~s})$ scans using only five angles would suffice. The calibration of the microscope was regularly checked before each series of measurements in order to reduce any background measurement of birefringence. The images were all generated using a $580 \mathrm{~nm}$ bandpass filter (10 $\mathrm{nm}$ half-height peak width).

The MetriPol microscope can measure the light-transmission variation as a function of the polarization of the incoming light by taking the analyser out of the light path and hence quantify and correct for any polarization-dependent scattering or absorption caused by the sample. In the early stages of this study, analysis was regularly performed without the analyser in the light path. In the event, it was found that the transmission variation was always negligible.

\subsection{Seeding experiments on glucose isomerase}

Glucose isomerase crystallization droplets were prepared and left to equilibrate for one to two weeks before execution of the seeding experiments. A cat's whisker was used for the seeding. For each seeding experiment, a control was set up, ensuring that the results obtained with seeding were not the consequence of dehydration of the droplets. The control experiments were carried out in the same way as the seeding but without the whisker being soaked in the precipitate beforehand.

\section{Results}

The different phases of both proteins were observed with the microscope. For the initial screen, a low-power objective $(4 \times)$ 

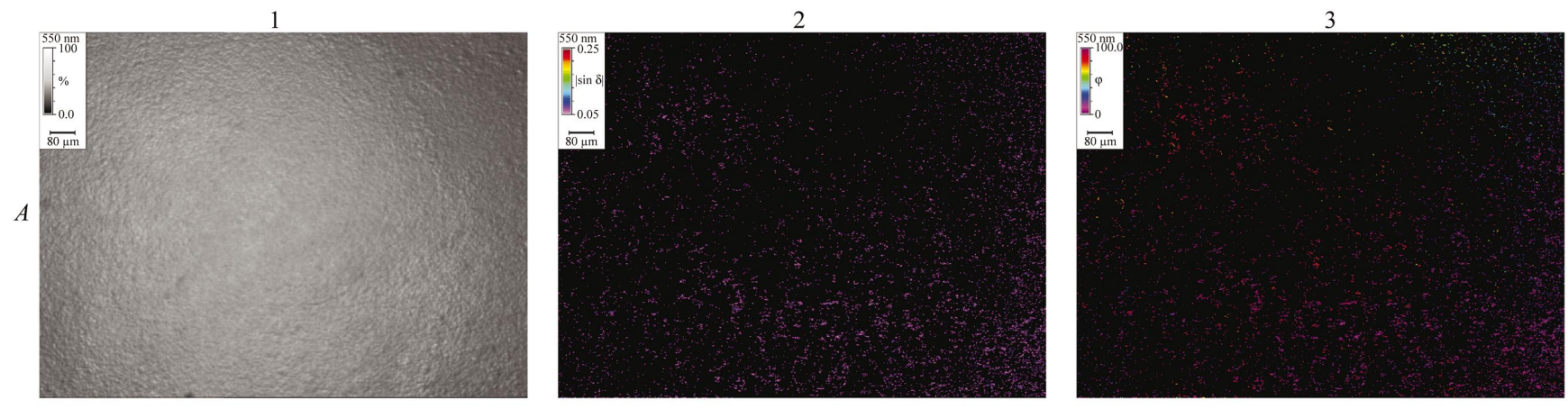

[AS]
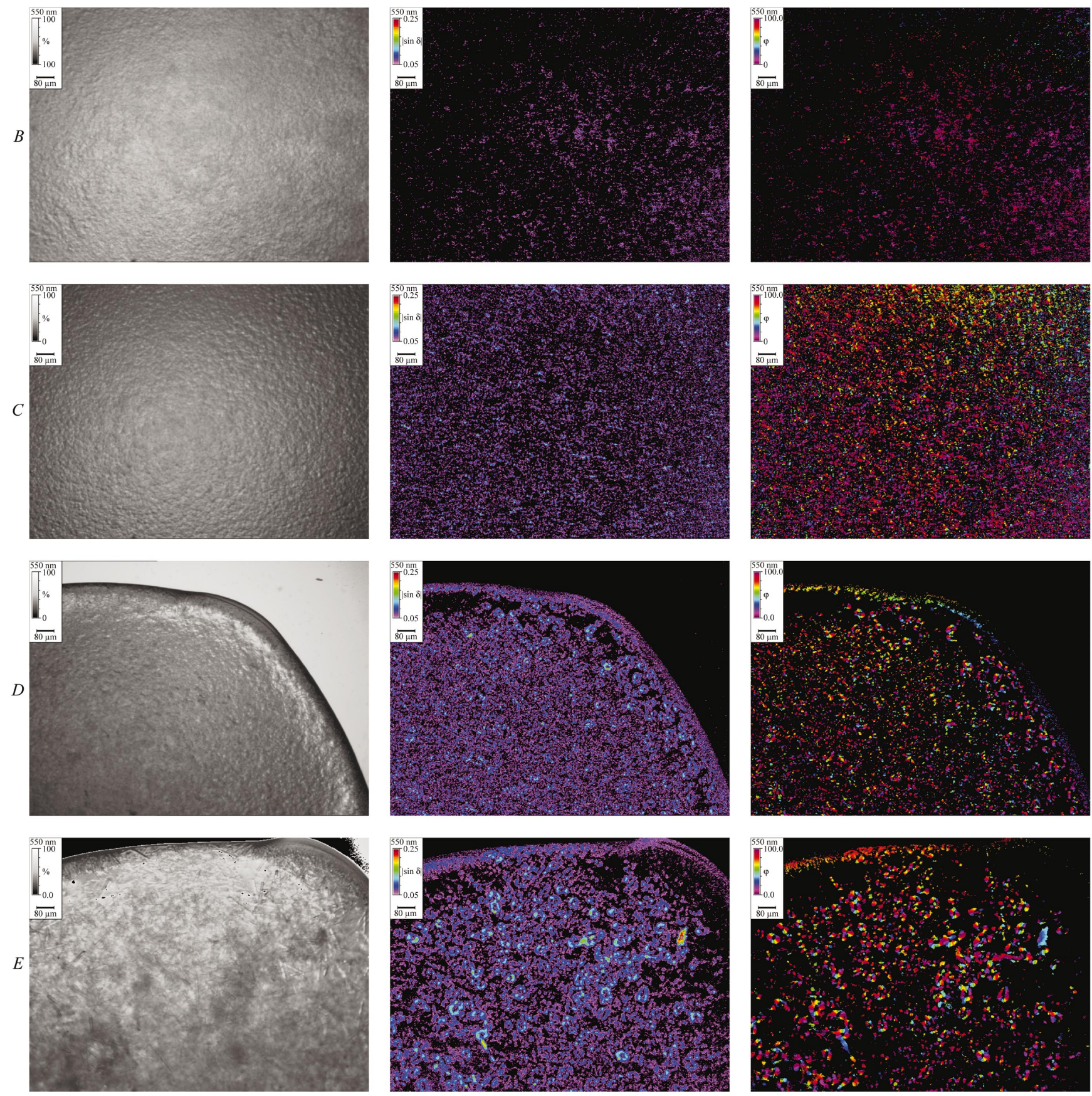

Figure 1

Images of glucose isomerase crystallization droplets. Column 1 shows the transmission images, column 2 the magnitude of birefringence and column 3 the orientation of the birefringence. The concentration of the crystallization agent (ammonium sulfate) was reduced through rows $A-E$. All the images were taken with a low-power magnification objective $(4 \times)$. The same threshold $(|\sin \delta|>0.05)$ was used for all images. 
was used in order to image as a large a part of the droplet as possible.

\subsection{Examination of crystallization conditions using standard non-polarized microscopy}

The glucose isomerase phase diagram with all parameters kept constant except for the precipitating agent concentration was determined. This led to the characterization of three easily distinguishable phases: precipitate (ammonium sulfate concentration $>1.6 \mathrm{M}$ ), crystals (initially as acicular spherulites, sometimes referred to as sea urchins; $1.5-0.8 \mathrm{M}$ ammonium sulfate) and single crystals (0.9-0.7 $M$ ) and soluble protein $(<0.7 M$ ammonium sulfate; corresponding to clear drops). The texture of the precipitate became more pronounced as the crystallization conditions were approached (Fig. 1; $A 1-E 1)$.

A similar phase sequence was determined for lysozyme in sodium chloride. A precipitate was visible for concentrations larger than 3.7 M. Clusters of acicular crystals were visible from 3.6 to $2.3 M$ sodium chloride. A mixture of acicular crystals and single crystals (tetragonal lysozyme crystals) were seen between 2.2 and $1.7 \mathrm{M}$, single crystals were seen from 1.6 to $0.5 M$ and clear droplets were seen at concentrations lower than $0.45 \mathrm{M}$.

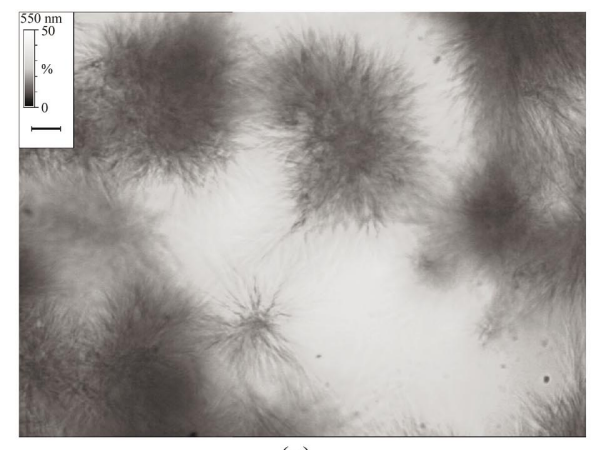

(a)

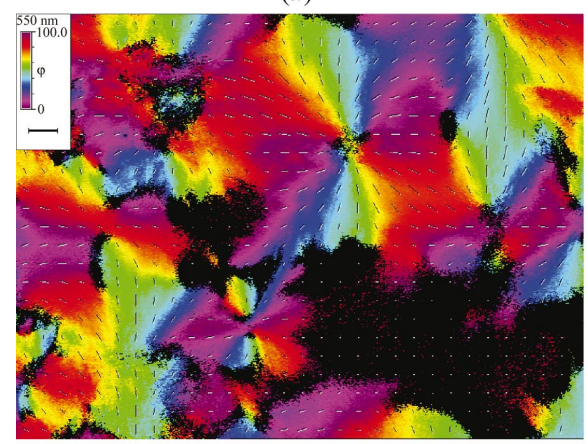

$(c)$

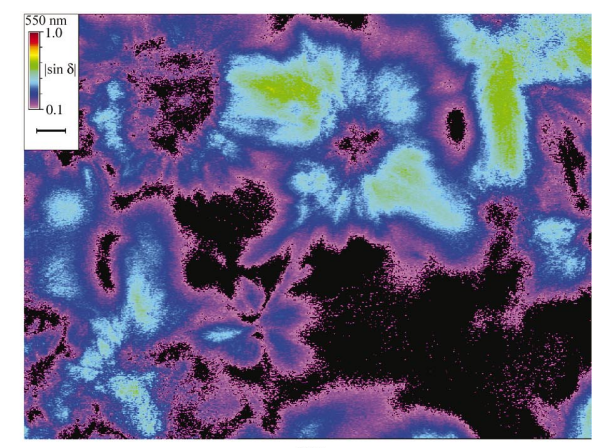

(b)

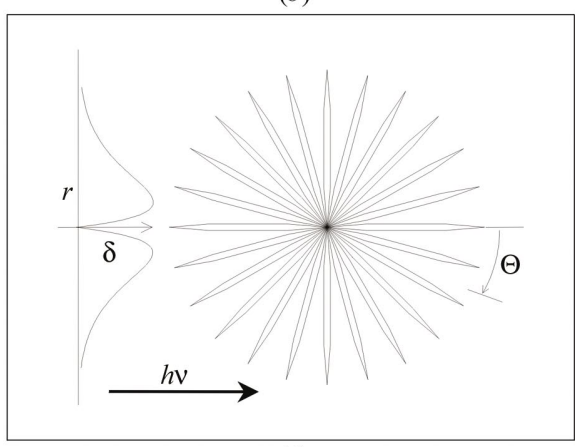

$(d)$
Figure 2

Characterization of the birefringent precipitate found for lysozyme. $(a-c)$ The transmission, $|\sin \delta|$ and $\varphi$ images produced with a medium-power objective $(20 \times)$ and a $|\sin \delta|$ threshold of 0.1 . The roughly annular features in the $|\sin \delta|$ image correspond to the identifiable acicular spherulites in the intensity image. The 'butterfly pattern' illustrates the symmetry of the alignment of the acicular crystals around the centre of the spherulite. The concentration of crystallization agent (sodium chloride) was $2.7 \mathrm{M}$ and the concentration of lysozyme was $75 \mathrm{mg} \mathrm{ml}^{-1}$. (d) Model of the birefringence caused by acicular spherulites. The light path is horizontally in the plane of the drawing. The birefringence varies symmetrically with increasing distance, $r$, to the centre of the spherulite. $\Theta$ is the angle between the viewing direction and the axis of any acicular crystallite.

\subsection{Examination of crystallization conditions using}

To facilitate the analysis of the birefringence microscopy measurements, a general threshold value was applied such that only areas with $|\sin \delta|>0.05$ were coloured; any other areas were masked out. This made any significantly birefringent features stand out more clearly. This threshold setting was also a useful way to reduce the noise in the orientation images. The default threshold of $|\sin \delta|>0.02$ applied by the program made it harder to distinguish the birefringent elements from the background birefringence measured by the setup.

In the glucose isomerase experiments the precipitate at high precipitant concentrations (3.8-2.4 $M$ ammonium sulfate) showed no significant birefringence (very low $|\sin \delta|<0.05$ comparable with the background readings; $A 2-A 3$ in Fig. 1) dicating no measurable order present. However, with creasing precipitant concentration (lower than $2.4 \mathrm{M}$ monium sulfate), the birefringence was seen to increase to point where small crystals became observable in the not shown).

The birefringence occurring as the precipitant concentration was reduced was of an annular appearance (D2-E2, D3E3 in Fig. 1) with a radial distribution of the slow extinction angle (we describe this as a 'butterfly pattern'). The birefringent areas corresponded to apparently non-crystalline globules in the transmission image.

\subsection{Identification of the birefringent globules as the acicular spherulite}

An initial coarse crystallization conditions matrix did not reveal the nature of these annular features and thus seeding experiments on glucose isomerase were performed in order to ascertain the crystalline nature of the precipitate. A finer matrix experiment showed transitions where both the annuli and obvious crystals were present in the same droplet.

The first (and as it later turned out incorrect) assumption was that the annuli were of a liquid crystal nature, i.e. one-dimensional or two-dimensional ordered crystals with little or no translation symmetry (liquid crystals such as liposomes sometimes show similar birefringence; Hyde, 2001). However, the finer matrix screen revealed acicular spherulites (Fig. 2a). Careful inspection reveals the close correspondence between the birefringence patterns seen using a high-power 
objective in Fig. 2 (lysozyme) and low-power objectives in E1E3 in Fig. 1 (glucose isomerase).

Packed crystallites of this type would be expected to show form birefringence. Looking vertically down through the centre of a 'sea urchin', an area of low birefringence is expected (Fig. $2 d$ ). For acicular crystallites, the birefringence then increases as the angle $\Theta$ between the crystallites and the light path increases until the density of crystallites starts to diminish. This is precisely what is observed.

To ease the interpretation of the orientation information in Fig. 2(c), we have overlaid the image with azimuthal lines corresponding to the colour information. It can be seen that these lines point radially away from a centre of nucleation.

The slow axis corresponds to the larger refractive index, located along the long axis of the packed crystallites. To analyse the sign of the birefringence, we approximate the spherulite to a collection of bundled rods radiating from the centre of the spherulite. We can deduce from Fig. 2(c) that the larger refractive index is along the unique axis, making the bundles positively birefringent. The sign of the form birefringence for bundles of rods is positive when the rods have a refractive index higher than the surrounding medium (4). This is consistent with our hypothesis that the birefringence we see related to the sperulites is a kind of form birefringence.

It is worth noting that the birefringence in the bottom row of Fig. 1 is reduced compared with the previous row, since the form birefringence is reduced while the crystals are still too thin to have any measurable intrinsic birefringence.

\section{Discussion}

We have shown that the rotating-polarizer technique is capable of detecting protein crystallites in what appears to be an amorphous precipitate, as can be seen in Fig. 3. If techniques such as that presented here come into general use, the drastic increase in the region of the phase diagram in which crystal growth can be detected will lead to reduced amount of

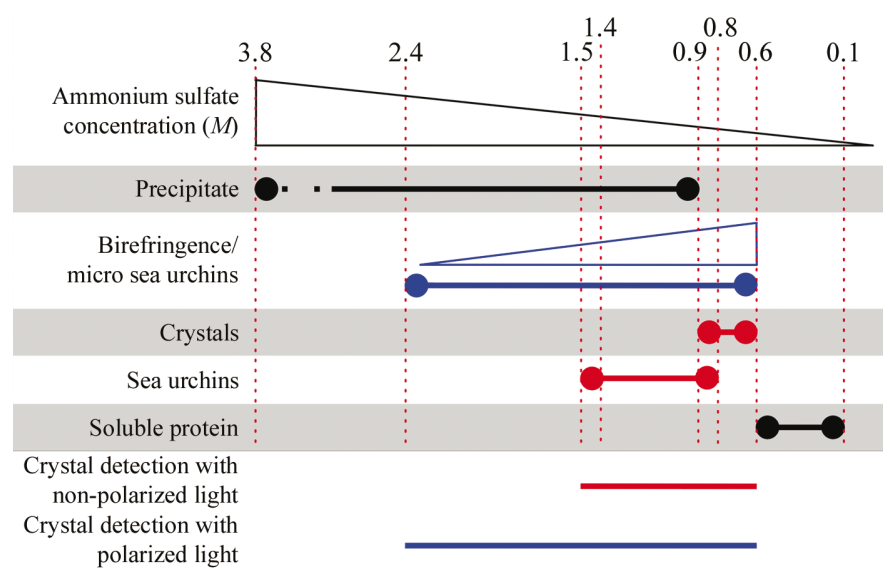

Figure 3

Comparison of glucose isomerase crystallization trials with/without use of the rotating-polarizer technique. Sensitive birefringence measurements led to crystallinity being detected in a region of the phase diagram that is twice as large as the region in which crystallinity could be detected by visual inspection. protein needed for the initial screening, since coarser screenings may be possible and crystal detection will become easier.

We chose the two proteins reported here for their ease of crystallization and availability and have surprisingly found two very similar crystallization patterns, with microscopic acicular spherulites appearing at precipitant concentrations higher than that needed for growing crystals suitable for X-ray diffraction. Whether this is a pure coincidence or whether it is always the case we cannot presently say. However, it is well known that crystallizing at increased concentration of precipitant leads to multiple nucleation sites (Bhamidi et al., 2001; Mushol \& Rosenberger, 1997). We may have accidentally come across an extreme instance where we see multiple nucleation sites each giving rise to multiple nucleations (Figs. 1 and 2). This needs to be studied further before any conclusions may be drawn.

The crystal/crystallite detection is strongly affected by birefringent contamination of the droplet by foreign material, which can result in false positives, and so it is unlikely that a $100 \%$ failsafe scoring would be achievable for a fully automated version. Likewise, droplets with strong phase separation and/or presence of skin will lead to light scattering and this will reduce the quality of the data. However, as mentioned, it is possible to eliminate scattering effects by repeating the measurements without the analyser in the light path (Björknäs et al., 2003). If only the birefringence measurements are used without reference to the transmission image, false negatives will occur for cubic crystals or crystals that are not birefringent along the viewing direction. Therefore, any development of the program towards full automation will have to include feature-recognition algorithms (Wilson $e t$ al., 2002) applied to the intensity image.

There is furthermore the issue of setting the correct threshold for detecting any indication of crystalline material in the droplets, while at the same time reducing the number of false positives. As stated, we found that only imaging the area of the samples for which the $|\sin \delta|$ values exceeded 0.05 emphasized the birefringent features over any background. However, for the detailed study of the character of the birefringence we used a higher threshold $(|\sin \delta|>0.1)$ to achieve a better contrast between the feature we were studying and any background. The threshold setting is purely of relevance in the presentation and analysis of the generated data and thus it is possible to start with a high threshold and then reduce it until the number of false positives becomes unacceptable.

On the basis of this study, a number of issues needs to be considered for future use of techniques such as this. The first is the lack of birefringence-free crystallization plates. We have tested a large number of so-called birefringence-free plastic crystallization plates, but they have all been shown to have background birefringence several magnitudes greater than the crystallites presented here. Others using related birefringence techniques (Bodenstaff et al., 2002) have had to make their own trays.

Once suitable trays have become available, the technique needs to be integrated with an $X Y$ stage to facilitate the data 
capture, which at the moment is a lengthy process with most of the time spent on positioning the sample in the light path.

Another issue is that at the moment the data analysis is performed visually for the individual wells. Clearly, it would be desirable if the software program performed at least a coarse scoring of the results, reducing the number of images that need to be inspected visually. This would be of particular advantage for use in high-throughput facilities.

We thank Dr Elspeth Garman of LMB, Oxford University for useful suggestions and helpful discussions. Special thanks are also due to Professor Mike Glazer of the Clarendon Laboratory, Oxford University for constructive input and valuable help with the manuscript. AE thanks EPSRC for a studentship. VF is a Royal Society University Research Fellow.

\section{References}

Bhamidi, V., Skrzypczak-Jankun, E. \& Schall, C. A. (2001). J. Cryst. Growth, 232, 77-85.
Björknäs, K., Geday, M. A. \& Raynes, E. P. (2003). Liq. Cryst. 30, 889-897.

Bodenstaff, E. R., Hoedemaeker, F. J., Knil, M. E., de Vrind, H. P. M. \& Abrahams, J. P. (2002). Acta Cryst. D58, 1901-1906.

Bragg, W. L. \& Pippard, A. B. (1953). Acta Cryst. 6, 865-867.

Carrell, H. L., Rubin, B. H., Hurley, T. J. \& Glusker, J. P. (1984). J. Biol. Chem. 259, 3230-3236.

Ducruix, A. \& Giegé, R. (1999). Editors. Crystallization of Nucleic Acids and Proteins. Oxford University Press.

Geday, M. A., Kaminsky, W., Lewis, J. G. \& Glazer, A. M. (2000). J. Microsc. 198, 1-9.

Glazer, A. M., Lewis, J. G. \& Kaminsky, W. (1996). Proc. R. Soc. London Ser. A, 452, 2751-2765.

Hyde, S. T. (2001). Identification of Lyotropic Liquid Crystalline Mesophases. In Handbook of Applied Surface and Colloid Chemistry. New York: John Wiley \& Sons.

Hartshorne, N. H. \& Stuart, A. (1969). Crystals and the Polarizing Microscope. London: Edward Arnold.

Jancarik, J. \& Kim, S.-H. (1991). J. Appl. Cryst. 24, 409-411.

Mushol, M. \& Rosenberger, F. (1997). J. Chem. Phys. 107, $1953-$ 1962.

Nollert, P. (2003). J. Appl. Cryst. 36, 1295-1296.

Oldenbourg, R. \& Ruiz, T. (1989). Biophys. J. 56, 195-205.

Wilson, J. (2002). Acta Cryst. D58, 1907-1914. 